\title{
Transport properties and structures of vortex matter in layered superconductors
}

\author{
M. F. Laguna, D. Domínguez and C. A. Balseiro \\ Centro Atómico Bariloche and Instituto Balseiro, Comisión Nacional de Energía Atómica, 8400 San Carlos de Bariloche, Río \\ Negro. Argentina
}

\begin{abstract}
In this paper we analyze the structure, phase transitions and some transport properties of the vortex system when the external magnetic field lies parallel to the planes in layered superconductors. We show that experimental results for resistivity are qualitatively consistent with numerical simulations that describe the melting of a commensurate rotated lattice. However for some magnetic fields, the structure factor indicates the occurrence of smectic peaks at an intermediate temperature regime.
\end{abstract}

\section{INTRODUCTION}

The discovery of high $T_{c}$ superconductivity renewed the interest for the thermodynamic, structural and dynamical properties of vortex matter. Due to the large temperatures available for the vortex system and an important number of relevant parameters, as anisotropy, disorder and the magnitude and direction of the external magnetic field, the physics of these systems is very rich. First and second order phase transitions between a low temperature solid phase an a high temperature liquid phase have been predicted and observed experimentally. The low temperature phase can be either a crystalline or a glassy phase depending on the ampunt and nature of the disorder present in the sample.t.

All the cuprate high $T_{c}$ materials have in common a crystalline structure based on the existence of $\mathrm{CuO}$ planes. This makes all of them anisotropic materials with a layered structure. In the usual convention, the $c$-axis points in the direction perpendicular to the planes and the $a$ and $b$ axis are the in-plane crystalline axis.

Concerning the study of the vortex properties in these materials, most of the work was devoted to the study of the configuration in which the external field points along the $c$-axis, particularly in the case of experimental work. There is however an increasing interest in the properties of the system for fields parallel to the planes 3 Theoretically, in many cases, the system is simplated as a highly anisotropic but homogeneous system.10 The planes however act as a strong potential for the vortices that tend to localize them between planes where superconductivity is weak.

In a clean system and at low temperatures, for the external field parallel to the $a b$-planes, the vortices form an anisotropy - distorted Abrikosov lattice. If the lattice is commensurate with the periodic potential due to the planes, the ground state of the system is a commensurate structure like the one schematically shown in Fig.(1a). In this particular case, the vortex density $\delta$ in the direction perpendicular to the planes is modulated with a period given by the lattice parameter of the vortex lattice. For this type of structures, the most salient theoretical prediction is the existence, at an intermediate temperature interval, of a smectic phase.6 In this picture the transition between the crystalline state and the high temper- ature liquid state takes place in two steps. In the intermediate regime the system develops long (or quasi-long) range order in the direction perpendicular to the planes, i.e. the liquid develops some density oscillations along this direction as a precursor of the frozen state. This phase is known as the smectic phase. The existence of the smectic phase is unconfirmed and there are many relevant questions concerning its nature and stability. One of them is what happens if the field does not produce a commensurate structure like the one shown in the figure. One possibility is that the lattice locally retains its structure and orientation and generates discommensuration to accommodate to the periodic potential generated by the planes. If the mismatch is not too large the discommensurations are far apart and the physical properties of the system are not very affected. In this case it could be possible to detect the existence of the smectic phase without tuning the external field to its commensurate value. The other alternative is that as the field is changed, the vortex lattice rotates and distorts - due to the anisotropic properties of the system- to form a new structure that is commensurate with the periodic potential like the one shown in Fig.(1b). In this paper we will show that at low fields, as the field changes, between two consecutive commensurate structures of the type of Fig.(1a), there are a number of commensurate phases of the type illustrated in Fig.(1b).

For the particular case of the rotated structure shown in the figure, the vortex density $\delta$ of the lattice is the same between any two consecutive planes. In this case, as the temperature increases, the lattice could melt going directly from the solid to the liquid state. If this is the case, it would be very difficult to observe the smectic phase for an arbitrary chosen external field.

The transport properties of the vortex system for the configuration of interest were recently measured 11 and the data were compared with the theory of the smectic phase. Experiments and theory are only partially consistent.

In the rest of the paper we discuss, in terms of the London theory with a periodic potential, the stability of the different commensurate phases. We also present some numerical simulations for the resistivity in layered structures and analyze the corresponding low temperature vortex configurations. We will show that the exper- 
imental results for the resistivity are qualitatively consistent with numerical simulations. For some fields, the structure factor indicates the occurrence of smectic peaks at an intermediate temperature regime.

(a)

(b)

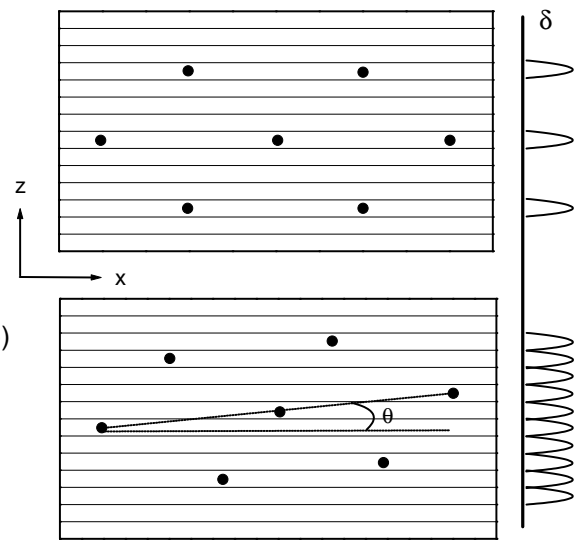

FIG. 1. Commensurate structures at low temperatures. In (a) the magnetic field applied in the $y$-direction generates a non-rotated vortex lattice for which the vortex density $\delta$ is modulated in the direction perpendicular to the ab-planes. In (b) the vortex lattice is rotated an angle $\theta$, and $\delta$ is the same for all the planes.

\section{COMMENSURATE STATES IN THE LONDON APPROXIMATION}

In order to analyze the low temperature structures of the vortex lattice we resort to the London approach in an anisotropic material and in the presence of a uniaxial potential representing the effect of the planes. Following the work by Campbell, Doria and Kogan 10 (CDK), the free energy per unit length in the direction of vortices for an anisotropic material is given by:

$$
F_{0}=\int\left(\mathbf{h}^{2}+\lambda^{2} m_{i j} \operatorname{curl}_{i} \mathbf{h} \operatorname{curl}_{j} \mathbf{h}\right) \frac{d x d z}{8 \pi}
$$

where $\mathbf{h}(x, z)$ is the local magnetic field in the plane perpendicular to the vortices, $\lambda^{2}$ is proportional to the $a v$ erage mass $M_{a v}=\left(M_{1} M_{2} M_{3}\right)^{1 / 3}$ with $M_{k}$ being the principal values of the mass tensor $M_{i j}$ and $m_{i j}=$ $M_{i j} / M_{a v}$ is the effective-mass tensor. We consider the external field in the $y$-direction (parallel to the planes) that coincides with one of the principal axis of the crystal, $\mathbf{h}=\left(0, h_{y}, 0\right)$. Then we take $m_{x x}=m_{y y}=m_{1}$ and $m_{z z}=m_{3}$.

For a vortex lattice, $h_{y}(x, z)$ is a periodic function with nonzero Fourier components $h_{y}(\mathbf{G})$, where $\mathbf{G}$ are the reciprocal lattice vectors of the vortex lattice. The free energy is minimized with respect to $\mathbf{h}$ and following CDK, $F_{0}$ is given by:

$$
F_{0}=\frac{B^{2}}{8 \pi} \sum_{\mathbf{G}} \frac{1}{1+\lambda^{2}\left(m_{1} G_{x}+m_{3} G_{z}\right)},
$$

where $B=\phi_{0} n$ is the magnetic induction, $\phi_{0}$ is the flux quantum and $n$ is the number density of vortices. In this expression, the summation is over all the reciprocal lattice vectors $\mathbf{G}$ of the vortex lattice. The mass anisotropy distorts the hexagonal lattice compressing it in the $z$-direction and expanding it along the $x$-direction. The lattice can rotate to form an angle $\theta$ with the $x$-axis as shown in Fig (1b). For this particular configuration, in which the magnetic field is parallel to one of the principal axis of the crystal, CDK shown that the $F_{0}$ contribution to the free energy does not depend on $\theta$.

The above considerations are valid for continuous anisotropic superconductors. In layered superconductors, the presence of planes introduces a periodic potential that will partially break the degeneracy in $\theta$. In a first order approximation, we describe the effect of the planes by including a periodic potential that tends to localize the vortices in the interplane spacing:

$$
F=F_{0}+\int h_{y}(x, z) V(z) d x d z
$$

where $V(z)$ is a periodic potential with the periodicity of the $c$-axis lattice parameter. The condition for commensurability of the vortex lattice with the periodic potential, corresponding to the situation in which all vortices are placed at a minimum of $V(z)$, is given by the condition $x_{j}=n s$ where $x_{j}$ is the $x$-component of the coordinate of vortex $j, n$ is an integer and $s$ is the $c$-axis lattice parameter (the interplane distance). In the reciprocal space this condition gives a relation between the reciprocal lattice vectors and the vector $\mathbf{Q}$ of the periodic potential $V(z)$ :

$$
\mathbf{Q}=\frac{2 \pi}{s} \hat{\mathbf{z}}=p \mathbf{G}_{\mathbf{1}}+q \mathbf{G}_{\mathbf{2}}
$$

where $p$ and $q$ are integers and

$$
\begin{gathered}
\mathbf{G}_{\mathbf{1}}=\frac{2 \pi}{\gamma L} \frac{\sin (\theta+\pi / 3)}{\sin \pi / 3} \hat{\mathbf{x}}-\frac{2 \pi \gamma}{L} \frac{\cos (\theta+\pi / 3)}{\cos \pi / 3} \hat{\mathbf{y}} \\
\mathbf{G}_{\mathbf{2}}=-\frac{2 \pi}{\gamma L} \frac{\sin \theta}{\sin \pi / 3} \hat{\mathbf{x}}+\frac{2 \pi \gamma}{L} \frac{\cos \theta}{\cos \pi / 3} \hat{\mathbf{y}}
\end{gathered}
$$

with $\gamma^{2}=\sqrt{m_{1} / m_{3}}$ the anisotropy factor and $L^{2}=$ $\left(2 \Phi_{0}\right) /(\sqrt{3} B)$.

This condition can be put in the form:

$$
\tan (\theta)=\frac{p \cos (\pi / 3)-q}{p \sin (\pi / 3)}
$$

and

$$
B=\frac{2 \phi_{0} \gamma^{2} \sin ^{2}(\pi / 3)}{\sqrt{3} s^{2}(p \sin (\theta+\pi / 3)-q \sin (\theta))^{2}}
$$

In Fig.(2), all angles $0 \leqslant \theta \leqslant \pi / 6$ satisfying the condition for commensurability are shown as a function of the magnetic induction $B$. In the London approximation, since 
the free energy is $\theta$ independent, all commensurate states obtained for a given magnetic induction $B$ are degenerate. As can be seen in Fig.(2), for low fields, between two consecutive commensurate structures of the type of Fig.(1a) - corresponding to $\theta=0$ or $\pi / 6$ - there are a large number of commensurate phases with $\theta \neq 0$. As the field increases the number of commensurate phases decreases and in the limit of high magnetic fields (or high anisotropy) there are only two undistorted lattices corresponding to $\theta=0$ and $\pi / 6$, and at all temperatures the vortex density is the same between any two consecutive planes. In this limit, increasing the magnetic field compresses the vortex lattice in the $z$-direction. Ow simple model is not appropriate to describe this limit 12 , 13

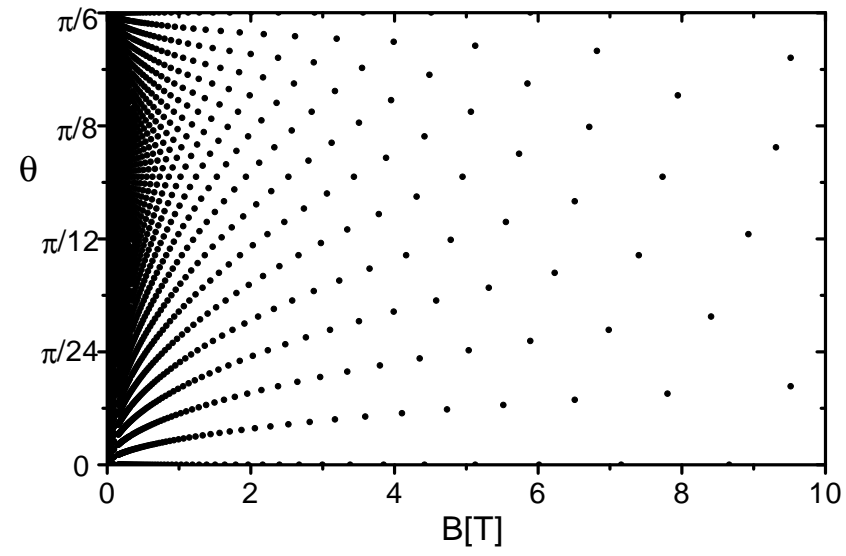

FIG. 2. Angles of rotation of the vortex lattice vs. magnetic field. Each point of the diagram correponds to a commensurate state of minimum energy. Magnetic field is in Tesla and the parameters are those typically used to describe YBaCuO: $s=10 \AA$ and mass anisotropy $m_{1} / m_{3}=50$.

For all angles, the high anisotropy generates structures where the vortex-vortex distance along the plane directions is much larger than the distance perpendicular to the planes, esiving rise to vortex chains. Recently $\mathrm{Hu}$ and Tachiki 1 studied the ground state configuration of the vortex lattice, using Monte Carlo simulations in the highly anisotropic XY model for large systems. They found structures consisting of buckling vortex chains. We interpret these results as rotated lattices with discommensurations due to the mismatch of the vortex lattice parameter with the interplane distance. In the numerical simulations, transverse discretization of the space could be another source of discommensurations. Although in general, we expect the discommensuration to form an angle of $45^{0}$ with respect to the planes, 44,15 the boundary conditions in a finite sample could stabilize discommensurations parallel to the planes. The buckling vortex chains structures are consistent with Bitter-pattern observations, however in the experimental case twin boundarips may be relevant to stabilize the observed structure.16

\section{NUMERICAL SIMULATIONS}

In this section we present numerical simulations for the transport properties and structure factor in an anisotropic system described by a three-dimensional (3D) Josephson-junction array. The model has been extensively used and is described in Refs. $[17,18]$ and here we give only a brief summary of the method.

The equilibrium physics of this system is described by the Hamiltonian of a three-dimensional frustrated XY model:192 21

$$
\begin{gathered}
H=-E_{J} \sum_{<i, i^{\prime}>\in a b-\text { plane }} \cos \left(\varphi^{i}-\varphi^{i^{\prime}}-A^{i i^{\prime}}\right)+ \\
\frac{1}{\gamma^{2}} \sum_{<i, i^{\prime}>\in c-\text { axis }} \cos \left(\varphi^{i}-\varphi^{i^{\prime}}-A^{i i^{\prime}}\right)
\end{gathered}
$$

with $E_{J}=\frac{\phi_{0}^{2} s}{16 \pi^{3} \lambda^{2}}(\lambda$ is the penetration length in the $a b$ planes and $s$ is the interplane distance) and $A^{i i^{\prime}} \equiv$ $(2 e / \hbar c) \int_{i}^{i^{\prime}} \mathbf{A} . d \mathbf{l}$ the integral of the vector potential of the external magnetic field from site $i$ to site $i^{\prime}$. The phases $\varphi^{i}(t)$ are defined in the nodes of a lattice and represent the phase of the order parameter. The thermodynamics of this Hamiltonian coincides with the equilibrium properties of the 3D Josephson-junction array.

The dynamics of the 3D Josephson-junction array is contained in the time evolution of the phases $\varphi^{i}(t)$. Nearest neighbor nodes are coupled with Josephson junctions characterized by critical currents $I_{c}^{i i^{\prime}}$ and normal resistances $R^{i i^{\prime}}$. The equations describing the model are

$$
\begin{gathered}
j^{i i^{\prime}}=I_{c}^{i i^{\prime}} \sin \left(\varphi^{i}-\varphi^{i^{\prime}}-A^{i i^{\prime}}\right)+ \\
\frac{\hbar}{2 e R^{i i^{\prime}}} \frac{\partial\left(\varphi^{i}-\varphi^{i^{\prime}}\right)}{\partial t}+\eta^{i i^{\prime}}(t) \\
\sum_{\left\{i^{\prime}\right\}} j^{i i^{\prime}}=j_{e x t}^{i}
\end{gathered}
$$

Equation (10) gives the current between the nearest neighbor nodes $i$ and $i^{\prime}$ with phases $\varphi^{i}$ and $\varphi^{i^{\prime}}$. Here $\eta^{i i^{\prime}}(t)$ is an uncorrelated gaussian noise that incorporates the effect of the temperature. Equation (11) ensures the current conservation at each node and $j_{e x t}^{i}$ is the external current applied at node $i$. Equations (10) and (11) are numerically integrated on time using a Runge-Kutta method. The typical time step used is $\Delta t=.1 \tau_{J}\left(\tau_{J}=\phi_{0} / 2 \pi R_{0} I_{c}\right)$ and the number of iterations is $20,000 \leq N \leq 100,000$. Details of the numprical method have been presented in previous works. 17.18 The mean in-plane critical currents $I_{c}^{\|}$are larger than the mean inter-plane critical currents $I_{c}^{\perp}$ by an anisotropy factor $\gamma^{2}\left(\gamma^{2} \equiv I_{c}^{\|} / I_{c}^{\perp}\right)$, and $I_{c}^{\|}=I_{c}=\frac{2 \pi E_{J}}{\phi_{0}}$. At the same time, the ratio between the in-plane resistance $R^{\|}$ and the out of plane resistance $R^{\perp}$ is given by $1 / \gamma^{2}$. We 
also consider a small amount of disorder by taking a uniform distribution of critical currents of width $\Delta$ defined as

$$
\Delta=\frac{\left(I_{c}^{\max }-I_{c}^{\min }\right)}{\left(I_{c}^{\max }+I_{c}^{\min }\right)}
$$

where $I_{c}^{\max }$ and $I_{c}^{\text {min }}$ are the maximum and minimum values of the critical current in the corresponding directions. The disorder simulated are typically $0 \leq \Delta \leq .1$. As in the previous section, we take the $z$-direction as the direction perpendicular to the planes (parallel to the $c$-axis of the crystal) and the external magnetic field along the $y$-direction. The magnetic fields simulated are $1 / 72 \leq f \leq 1 / 6$, where $f=B a^{2} / \phi_{0}$ ( $a$ is the square lattice period). The values of anisotropy are $1 \leq \gamma^{2} \leq 45$ and typical system sizes are $8 \leq L_{x}, L_{y}, L_{z} \leq 64$. We calculate the resistivity in the three directions by applying a small probe current and evaluating the average voltage. For example, for the resistivity $\rho_{\mu}$ in the $\mu$-direction we drive the system with a small current $I_{\mu}\left(I_{\mu}=0.01 I_{c}^{\mu}\right)$ and measure the voltage

$$
V_{\mu}=\frac{\hbar}{2 e}\left\langle\frac{d}{d t}\left(\varphi^{i+\mu}-\varphi^{i}\right)\right\rangle
$$

Then $\rho_{\mu}=V_{\mu} / I_{\mu}$.

\section{A. Transport properties}

The results for the resistivity calculated with periodic boundary conditions (PBC) along the field direction and free boundary conditions $(\mathrm{FBC})$ in the other directions are shown in Fig.(3). These results correspond to a sample with $f=1 / 12, \gamma^{2}=20, L_{x}=L_{y}=L_{z}=30, \Delta=.05$ and $N=50,000$. We found similar results for systems with $f=1 / 48,1 / 24,1 / 6, \gamma^{2}=5,10,25$ and the same sizes and disorder as the ones indicated above. The general behavior is qualitatively similar to the experimental data obtained by Grigera et al. shown in the inset for comparison.

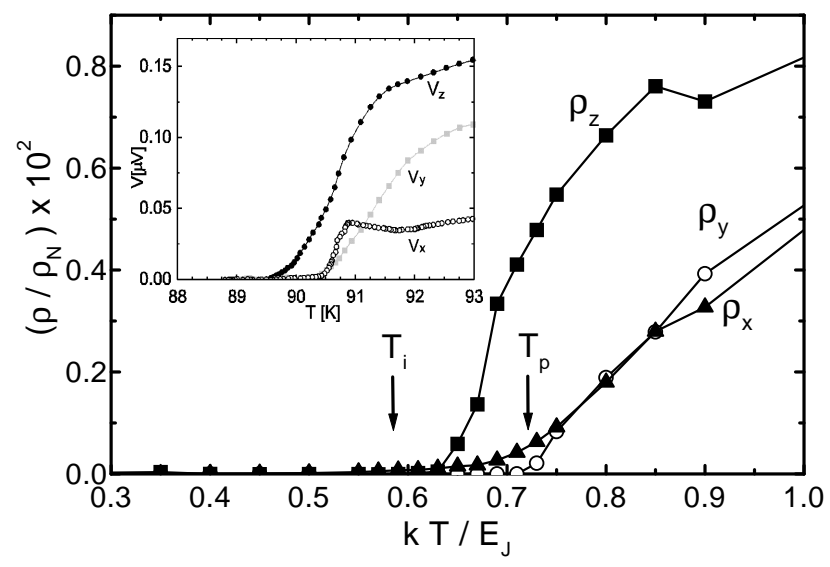

FIG. 3. Resistivities in the three directions for a system with FBC in $x$ and $z$ directions and $\mathrm{PBC}$ in the field direction. The parameters are $f=1 / 12, \gamma^{2}=20, \Delta=.05, N=50,000$ and $L_{x}=L_{y}=L_{z}=30 . \rho_{y}$ is the resistivity parallel to the field, $\rho_{x}$ is the perpendicular to the field but parallel to the ab-planes and $\rho_{z}$ is perpendicular both to the planes and the field. In the inset experimental results of Ref.[11] are shown.

The response of the system when the external current is perpendicular to the planes (and the Lorenz force is parallel to them) is given by $\rho_{z}$. For this geometry, the pinning due to the planes is unimportant and for temperatures higher than a characteristic temperature $T_{i}$ the resistivity $\rho_{z}$ increases rapidly. The stable phase for $T>T_{i}$ corresponds to a liquid phase and the rapid increase of $\rho_{z}$ is an indication of the high mobility of vortices parallel to the planes. The transition from the low temperature phase to the high temperature liquid phase is continuous. These results, obtained in the finite system, are not conclusive on whether the change of behavior observed at $T_{i}$ is a second order phase transition or a crossover between two different regimes. In finite systems, thermal activation is observed down to low temperatures and finite size scaling is necessary in order to characterize the transition. Based on previous results and the discussion of next section, we will refer to this behavior as continuous transition and to zero resistivity when the noise in the voltage is larger than its mean value, corresponding typically to $\rho_{\mu}<R_{\mu} \times 10^{-5}$, where $\mu=x, y, z$.

When the external current is applied along the $x$-direction, the Lorenz force is perpendicular to the planes and for temperatures $T \gtrsim T_{i}$ the in-plane pinning dominates the vortex dynamics resulting in a small $\rho_{x}$. The resistivity $\rho_{x}$ decreases strongly near a characteristic temperature $T^{*}>T_{i}$ and has a tail that extends down to $T_{i}$. This behavior is obtained for different values of the anisotropy and magnetic field, it qualitatively reproduces the experimental observation and resembles the prediction of the smectic phase theory. In this theory, as the temperature is lowered, the system undergoes a transition to a smectic phase at a temperature $T_{s}$. In the temperature interval $T_{i}<T<T_{s}$ the system develops long ( or quasi-long) range order in the direction perpendicular to the planes and the resistivity shows, in the vicinity of the smectic temperature $T_{s}$, a critical behavior of the form $\rho_{x}(T)-\rho_{x}\left(T_{s}\right) \propto\left|T-T_{s}\right|^{1-\alpha}$ where $\alpha$ is the specific heat critical exponent. At the temperature $T_{i}$, long range order in the direction parallel to the planes appears giving rise to a crystalline structure. Since in finite size systems the resistivity is not necessarily a good quantity to characterize a phase transition (in particular it will not show an infinite slope) we search for some signature of a smectic phase by analyzing the structure of the liquid for temperatures $T \gtrsim T_{i}$ corresponding to the regime where $\rho_{x}$ shows a tail. The details of the structure factors calculated with PBC at different temperatures are presented in next section.

Finally, we have also calculated the resistivity $\rho_{y}$ corre- 
sponding to a transport current along the field direction for which there is zero Lorenz force. In this geometry, the resistivity goes to zero at a temperature $T_{p}>T_{i}$. This behavior is due to finite size effects; dissipation occurs when vortices entangle to form a structure that percolates in the directions perpendicular to the external field. This happens at a temperature $T_{p}$ that is sensitive to the thickness $\left(L_{y}\right)$ of the sample the thicker the sample, the lower is $T_{p}$. Previous studies 18 of the resistivity in the direction of the field show that in the thermodynamic limit $T_{p}$ coincides with $T_{i}$ as experimentally observed and we expect the resistivity along the three directions to vanish at the same temperature.

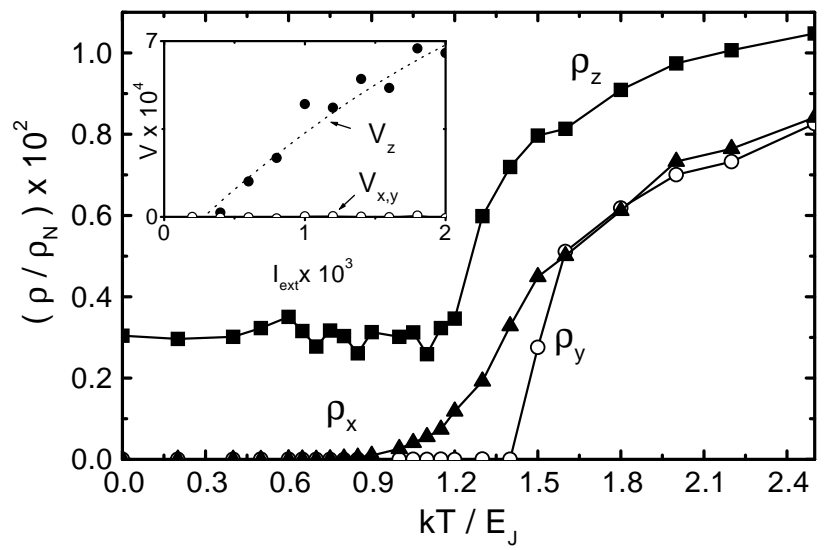

FIG. 4. Resistivities in the three directions with PBC for a system with $f=1 / 24, \gamma^{2}=9, L_{x}=L_{z}=48$ and $L_{y}=8$. $\rho_{x}, \rho_{y}$ and $\rho_{z}$ are defined in Fig.(3). In the inset the I-V characteristics for the current along the three directions are shown.

In order to discard boundary effects due to surface barriers, we have also calculated the resistivities and structure factors using $\mathrm{PBC}$ in the three directions. Results for the resistivity in a highly anisotropic sample with weak disorder are shown in Fig.(4). For this case, the parameters are $f=1 / 24, \gamma^{2}=9, L_{x}=L_{z}=48$, $L_{y}=8 \Delta=0$ and $N=30,000$. We have also done some simulations with $\mathrm{PBC}$ in the three directions for $f=1 / 72,1 / 48,1 / 8, \gamma^{2}=1-40$ and $N=10^{4}-10^{5}$, and we found similar results. While $\rho_{x}$ and $\rho_{y}$ behave essentially as in the previous case, as the temperature decreases $\rho_{z}$ saturates at a value different from zero, which increases with anisotropy. The I-V characteristics for the current along the three directions at low temperatures are shown in the inset and indicates a very small value of the critical current in the $z$-direction. The saturation of the resistivity at low temperatures is due to the fact that the run was done with a value of the transport current $j_{\text {ext }}$ larger than the critical current $j_{c}$ and the results correspond to a flux flow regime. A systematic study of the resistivities with $j_{\text {ext }}<j_{c}$ requires much more statistics and generates larger numerical errors. In any case, these results show two important points: first, for the systems under consideration, $\rho_{x}$ and $\rho_{y}$ are not very sensitive to the boundary conditions, and second, when vortices flow parallel to the planes, pinning is very weak. In the case of FBC the surface barriers generate some extra pinning that increases the critical current. In the thermodynamic limit, any rigid structure is pinned by impurities and surface effects and in this sense, it may be more appropriate to compare the case of $\mathrm{FBC}$ with experiments if results with $j_{\text {ext }}<j_{c}$ are not available for the case of PBC. As a side comment, notice that the noise in $\rho_{z}$ disappears at a temperature of the order of $T_{i}$ at which $\rho_{y}$ vanishes. A simple interpretation of this effect is that in the flux flow regime an ordered structure generates less noise than a liquid. However a systematic study of the noise, that could give information on the nature of the different phases, is needed to reach definitive conclusions.

\section{B. Structure factor}

In this section we present results for the structure factor calculated for different temperatures. At high temperatures, in the liquid phase, the system is highly disordered and presents vortex loop excitations. An instantaneous picture of the vortices crossing a plane perpendicular to the external field ( $\left.y=L_{y} / 2\right)$ is shown in Fig. (5a) for the same system as Fig.(4).

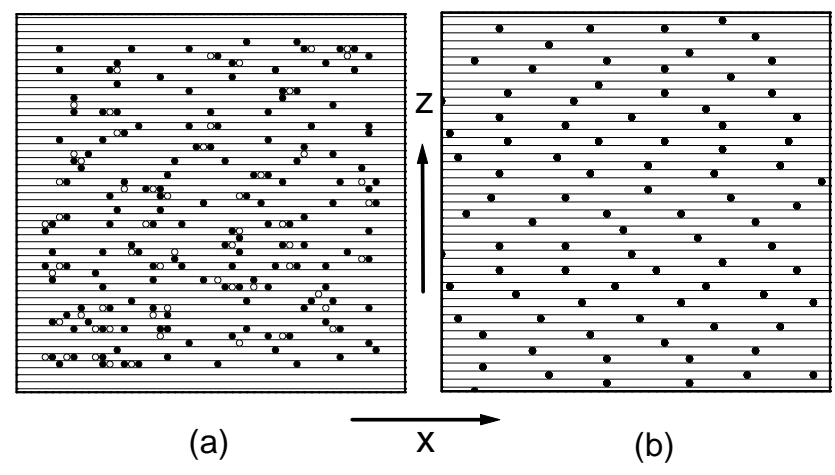

FIG. 5. Instantaneous configuration of the vortices in a plane perpendicular to the field for the system of Fig.(4). Black dots are vortices and white ones are antivortices. In (a) the temperature is $\frac{k T}{E_{j}}=1.1$ and in (b) $\frac{k T}{E_{j}}=0$.

The vortex-antivortex pairs are small loops confined between two $a b$-planes and cut by the $y=L_{y} / 2$ plane. As the temperature decreases the system evolves towards an ordered solid structure. It is known that, due to numerical limitations, in three dimensions it is very difficult to cool the system into an ordered lattice. The type of structures obtained by slowly cooling the system is shown in Fig (5b). We obtain this kind of structures for systems with $f \leq 1 / 12$. For higher magnetic fields, a triangular vortex lattice was found like the one predicted by Ref.[3]. 
Although the obtained structure is very disordered, the tendency to form vortex chains (at approximately $45^{0}$ in the figure) can be observed. This tendency is reflected by the structure factor $S\left(\mathbf{q}_{\perp}, y\right)$ defined as 22 :

$$
S\left(\mathbf{q}_{\perp}, y\right)=\frac{1}{N^{2}}\left|\sum_{j} \eta_{j} e^{i \mathbf{q}_{\perp} \cdot \mathbf{r}_{j}}\right|^{2}
$$

where $N$ is the total number of vortices, $\eta_{j}$ is the vortex charge: 1 for vortices and -1 for antivortices, and $\mathbf{r}_{\mathbf{j}}$ are the vortex coordinates in the direction perpendicular to the external field at a plane with coordinate $y$. We define $S\left(\mathbf{q}_{\perp}\right)$ as the average over $y$ of $S\left(\mathbf{q}_{\perp}, y\right)$.

In Fig.(6) the high and low temperature structure factor for the system of Fig.(5) are shown.

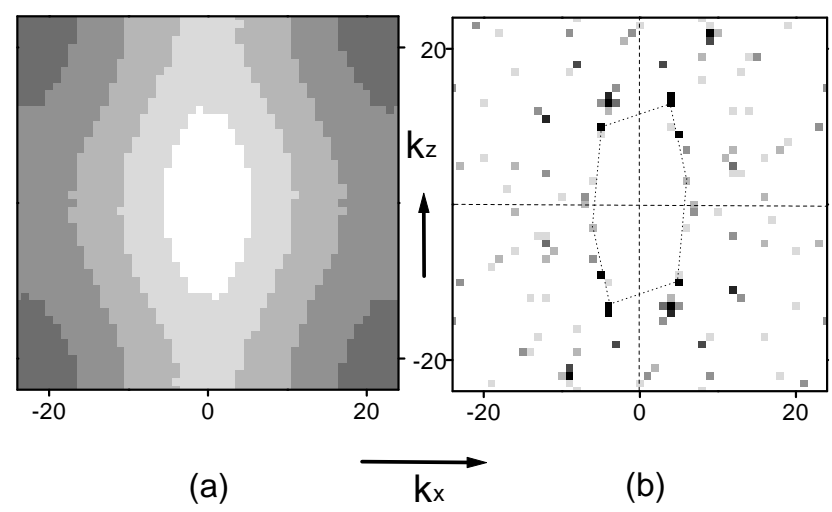

FIG. 6. Structure factor of the configurations of Fig.(5). (a) and (b) correspond to the same temperatures showed in that figure.

At high temperatures only a background of the form $\cos \left(q_{x} a\right)$, where $a$ is the lattice parameter of the junction network, is obtained. This background is due to the presence of small loops which in general are confined between to consecutive $a b$-planes, i.e. vortex-antivortex pairs which are oriented in the $x$-direction and bound at a distance $a$. At low temperatures, the background disappears and well defined peaks are observed. The peaks in the structure factor correspond to a rotated vortex lattice with a small angle $\theta$. In Fig.(6b) we draw one of the corresponding rotated hexagonal reciprocal space unit cell. We also see that there are peaks corresponding to the same lattice structure reflected in $180^{\circ}$ with respect to the $z$-axis. The coexistence of these two reflected structures could explain the chain-like ordering observed in real space configurations (Fig.(5b)). For this particular value of the external field, we follow the evolution of the structure factor as the system is cooled and observe some indications of a smectic phase at intermediate temperatures. A sequence of the structure factor at intermediate temperatures is shown in Fig.(7).

At temperatures $T \gtrsim T_{i}$, where the resistivity $\rho_{x}$ presents a small tail, a peak at $\mathbf{q}=\left(0, q_{y}^{0}\right)$ is clearly observed. This peak indicates the presence of a smectic phase. As the temperature is lowered from high temperatures, first the intensity of the smectic peak increases, goes through a maximum and then decreases as the crystalline peaks corresponding to a rotated structure increase. The wave vector $q_{y}^{0}$ may also be weakly temperature dependent: The first smectic peak observed has $q_{y}^{0} \simeq Q / 3(Q=2 \pi / s)$ and as the temperature is lowered it shift towards $Q / 2$. In Fig.(8) the temperature dependence of the smectic peak and one of the crystalline peaks are shown. The oscillations in the amplitude of the smectic peak are probably due to finite size effects, since only some discrete values of $q_{y}^{0}$ are consistent with the PBC. At temperatures $T \sim T_{i}$ both the smectic and the crystalline peaks coexist, indicating the coexistence of the two phases.

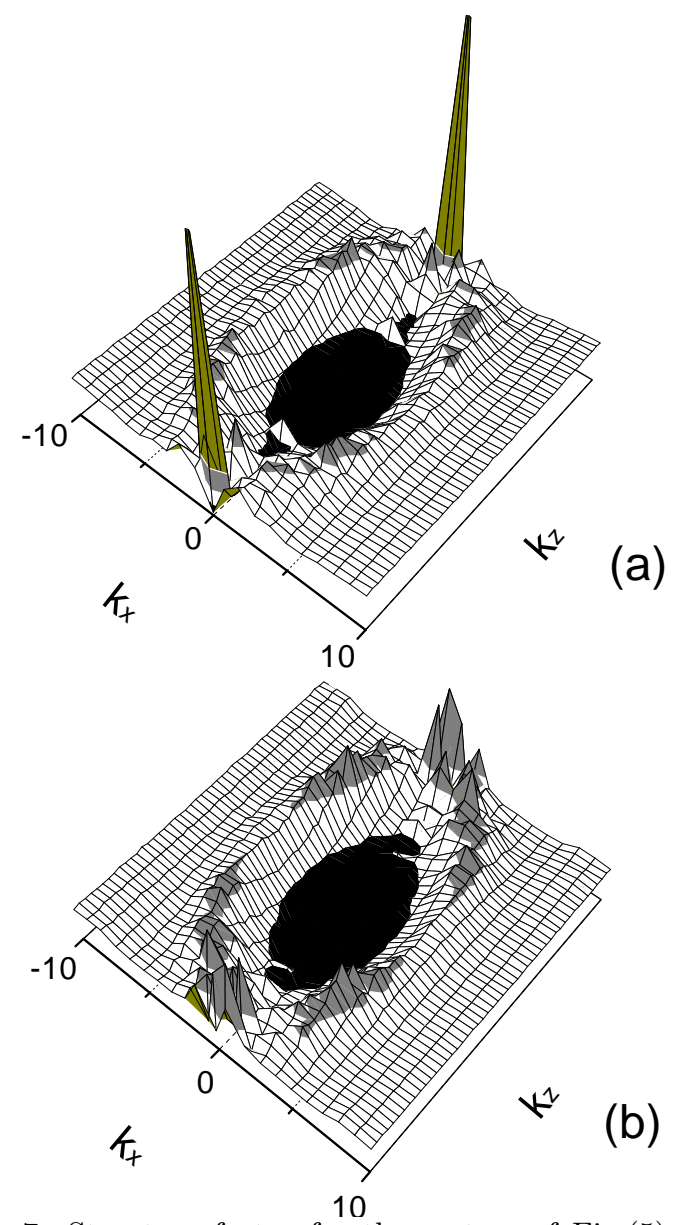

FIG. 7. Structure factor for the system of Fig.(5) at intermediate temperatures. A smectic peak can be observed at $q_{y}^{0} \simeq Q / 2$ at $T=0.53(\mathrm{a})$, which decreases at a lower temperature $\mathrm{T}=0.30(\mathrm{~b})$.

Since the symmetry group of the smectic phase is not a subgroup of the one corresponding to the symmetry of the rotated lattice, we expect a first order transition between these two phases. This is consistent with the observation of a coexistence of the two phases in the nu- 
merical simulations.

For other values of the parameters studied, $(f=1 / 24$ and $\left.\gamma^{2}=2.56,25\right)$, for which the low temperature phase corresponds to a rotated crystal with a larger rotation angle, we do not observe indications of a smectic order in the liquid phase. In these cases, our preliminary results indicate a continuous transition from the liquid to the frozen state without any precursor of long or quasi long range order in the direction perpendicular to the planes.

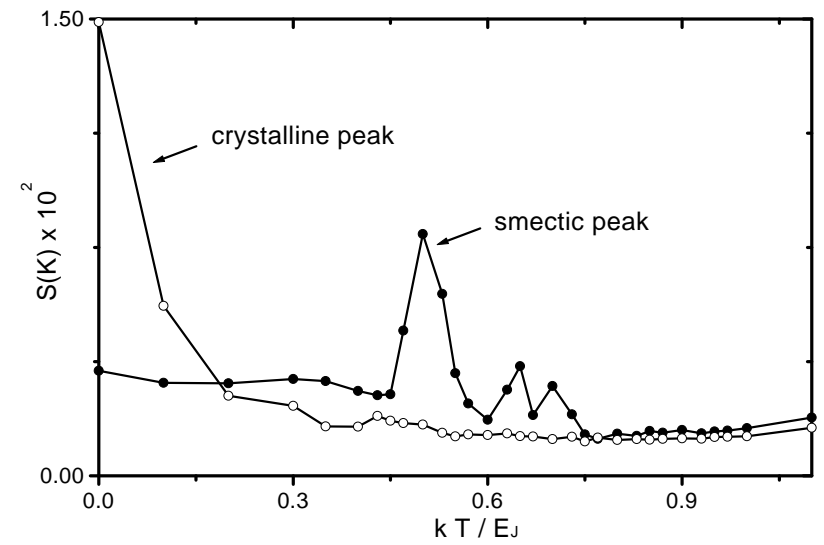

FIG. 8. Temperature dependence of the intensity of the smectic peak described in the text (open symbols) and a crystalline peak, $\left(q_{x}^{0}, q_{y}^{0}\right) \simeq(Q / 12, Q / 4)$ (filled symbols).

\section{SUMMARY AND DISCUSSION}

We have studied the problem of the low temperature structure, the thermodynamic and transport properties of vortices when the external field is applied parallel to the $\mathrm{CuO}$ planes in high $T_{c}$ superconductors. A simple analysis based on the London theory in the presence of a periodic potential indicates the possibility of a variety of commensurate structures that essentially correspond to anisotropy-distorted Abrikosov lattices rotated to match the periodic potential. For low fields a large number of degenerate structures corresponding to different rotation angles are obtained. Using parameters like those typically used to describe $\mathrm{YBaCuO}, s=10 \stackrel{\circ}{A}$ and a mass anisotropy $m_{1} / m_{3}=50$, for magnetic induction between 5 and 6 Tesla, we obtain about seven rotation angles which generate commensurate structures.

The numerical simulations in a XY-model clearly show the tendency to form rotated structures. Our results shows structures with grains of twin phases.

The transport properties qualitatively reproduce the experimental results. In particular, $\rho_{x}$ shows a rapid decrease and a tail that extends down to $T_{i}$. This behavior is similar to the predictions of the smectic phase theory although transport properties seem not to be enough to prove the existence of this phase at an intermediate temperature range.
When the rotated angle of the low temperature structure, which depends both on the value of the external field and the anisotropy, is small, we observe well defined peaks corresponding to a smectic phase. The vector $\mathbf{Q}$ and the amplitude of the smectic peak are temperature dependent and a first order transition is expected from the smectic to the crystalline phases. For the parameters that give a larger rotation angle of the crystalline phase, we observe a direct evolution of a system from the anisotropic liquid to the vortex lattice without any indication of an intermediate smectic phase.

\section{ACKNOWLEDGMENTS}

We acknowledge stimulating discussions with E.A. Jagla and E. Osquiguil, and thank S. Grigera for the data of Fig.(3). One of us (M.F.L.) acknowledge support from program FOMEC. We also acknowledge financial support from CONICET, CNEA, ANPCyT and Fundación Antorchas.

${ }^{1}$ G. Crabtree and D. Nelson, Physics Today 50, 38, April 1997.

${ }^{2}$ G. Blatter et al., Rev. Mod. Phys. 661125 (1994).

${ }^{3}$ L.N Bulaevskii and J.R. Clem, Phys. Rev. B 4410234 (1991).

${ }^{4}$ B. I. Ivlev and L. J. Campbell, Phys. Rev. B 4714514 (1993).

${ }^{5}$ W.K. Kwok et al., Phys Rev. Lett. 72, 1088 (1994).

${ }^{6}$ L. Balents and D.R. Nelson, Phys. Rev Lett. 73, 2618 (1994).

${ }^{7}$ L. Balents and D.R. Nelson, Phys. Rev. B 52, 12951 (1995).

${ }^{8}$ X. Hu and M. Tachiki, Phys. Rev. Lett. 804044 (1998).

${ }^{9}$ P. Olsson and P. Holme, cond-mat 9907118.

${ }^{10}$ L.J. Campbell et al., Phys. Rev B 38, 2439 (1988).

${ }^{11}$ S.A. Grigera et al., Phys. Rev. B 59, 11201 (1999).

${ }^{12} \mathrm{Hu}$ and Tachiki (unpublished).

${ }^{13}$ B.I. Ivlev, N.B. Kopnin and V.L. Pokrovsky, J. Low Temp. Phys. 80, 187 (1990).

${ }^{14}$ V.L Pokrovsky and A.L Talapov, Phys Rev. Lett 42, 65 (1979); Sov. Phys. JETP 51, 134 (1980).

${ }^{15}$ P. Martinolli et al., Helvetica Physica Acta, Vol. 55, 655 (1982).

${ }^{16}$ G. J. Dolan F. Holtzberg, C. Field and T. R. Dinger, Phys. Rev. Lett. 622184 (1989).

${ }^{17}$ D. Domínguez, N. Grønbech-Jensen and A.R. Bishop, Phys. Rev. Lett. 75, 717 (1995); Phys. Rev. Lett. 75, 4670 (1995); Phys. Rev. Lett. 78, 2644 (1997).

18 E.A. Jagla and C.A. Balseiro, Phys. Rev. B 53, R538 (1996); Phys. Rev. B 53, 15305 (1996).

19 R.E. Hetzel et al., Phys. Rev. Lett. 69518 (1992).

${ }^{20}$ Y.-H. Li and S. Teitel, Phys. Rev. Lett. 66, 3301 (1991). 
${ }^{21}$ X. Hu, S. Miyashita and M. Tachiki, Phys. Rev. Lett. 79 4044 (1997).

${ }^{22}$ Y.-H. Li and S. Teitel, Phys. Rev. B 47, 359 (1993).

${ }^{23}$ M. Tachiki and S. Takahashi, Solid State Commun. 70, 291 (1989). 